\title{
Association of Chronic Kidney Disease with Preserved Ejection Fraction Heart Failure Is Independent of Baseline Cardiac Function
}

\author{
Thomas A. Mavrakanas ${ }^{a, b} \quad$ Aisha Khattak $^{a, c} \quad$ Wei Wang $^{d}$ \\ Karandeep Singh ${ }^{a}$ e David M. Charytan ${ }^{a}$ \\ ${ }^{a}$ Renal Division, Brigham and Women's Hospital, Harvard Medical School, Boston, MA, USA; \\ ${ }^{b}$ Department of Medicine, Geneva University Hospitals, Geneva, Switzerland; ' ${ }^{\mathrm{C}}$ Division \\ of Renal Disease and Hypertension, Department of Internal Medicine, University of Texas \\ Health Science Center, Houston, TX, USA; d Department of Medicine, Brigham and Women's

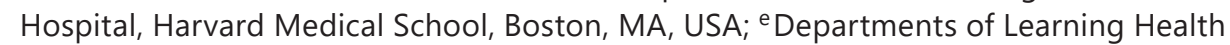 \\ Sciences and Internal Medicine, University of Michigan Medical School, Ann Arbor, MI, USA
}

\section{Keywords}

Chronic kidney disease $\cdot$ Heart failure with preserved ejection fraction · Left ventricular ejection fraction - Left ventricular mass index

\begin{abstract}
Background/Aims: Chronic kidney disease (CKD) is common among patients with heart failure with preserved ejection fraction (HFpEF) and is associated with worse clinical outcomes. This study aims to identify whether the association of CKD with HFpEF is independent of underlying echocardiographic abnormalities. Materials: We conducted a retrospective cohort study including patients without prevalent heart failure referred for echocardiography. Patients with serial echocardiograms, baseline left ventricular ejection fraction (LVEF) $\geq 50 \%$ and estimated glomerular filtration rate (eGFR) $\geq 90 \mathrm{~mL} / \mathrm{min} / 1.73 \mathrm{~m}^{2}$ were matched $1: 1$ with patients with eGFR $<60 \mathrm{~mL} / \mathrm{min} / 1.73 \mathrm{~m}^{2}$ for age ( \pm 5 years), sex, history of hypertension or diabetes, use of renin-angiotensin inhibitors, and LVEF $( \pm 5 \%)$. A secondary analysis included patients with preserved LVEF and normal left ventricular mass index matched for the same parameters except use of renin-angiotensin inhibitors. Results: Patients with CKD were at increased risk for HFpEF admission: crude hazard ratio (HR) 1.79 (95\% confidence interval [CI] $1.38-2.32, p<0.001$ ) and adjusted HR (for coronary disease, loop diuretics, left atrial diameter) 1.64 (95\% Cl 1.22-2.21, $p=0.001)$. LVEF and left ventricular diameter decreased over time in both groups but no difference was observed in rate of dropping. Results were similar in the secondary analysis (crude HR 1.99, 95\% Cl 1.07-3.71, $p=0.03$ and HR adjusted for left atrial
\end{abstract}


diameter $1.98,95 \% \mathrm{Cl} 1.05-3.75, p=0.04)$. Rate of change was similar for LVEF, pulmonary artery pressure, and left ventricular mass index in both groups. Conclusion: CKD is independently associated with incident HFpEF despite a similar change in relevant echocardiographic parameters in patients with or without CKD.

\section{Introduction}

Heart failure with preserved ejection fraction (HFpEF) refers to patients who have symptoms and signs of heart failure associated with preserved systolic function of the left ventricle, typically defined as a left ventricular ejection fraction (LVEF) $>50 \%$ [1]. HFpEF accounts for more than $40 \%$ of patients with heart failure [2]. Although the pathophysiology of HFpEF has not been fully elucidated, according to the traditional model, hypertension and myocardial ischemia are its major determinants [3, 4], through a pathway that involves concentric hypertrophy and diastolic dysfunction of the left ventricle, followed by left atrial and pulmonary venous hypertension and finally by right ventricular dysfunction as the last link in the chain [5]. Conversely, an emerging pathophysiological model proposes a novel paradigm for HFpEF shifting attention to endothelial inflammation, oxidative stress, and myocardial fibrosis $[6,7]$.

Chronic kidney disease (CKD) appears to play an important role in the pathogenesis of HFpEF [8]. In one prospective cohort study of $299 \mathrm{HFpEF}$ patients, for example, CKD was independently associated with worse cardiac mechanics, and worsening estimated glomerular filtration rate (eGFR) was associated with higher incidence of death or cardiovascular hospitalization [8]. In other studies, lower baseline eGFR has been associated with higher mortality and hospitalization from any cause in the setting of $\operatorname{HFpEF}[9,10]$.

Given these studies, it has been suggested that CKD is an underlying mechanism for the development of HFpEF' and that kidney dysfunction is responsible for impaired salt and water balance in an attempt to explain volume overload in HFpEF [11]. However, despite epidemiological data suggesting that low eGFR and high albumin excretion are independently associated with incident heart failure [12] or with HFpEF [13], we are unaware of any studies that have examined whether this association primarily relates to the high prevalence of echocardiographic abnormalities and underlying cardiac dysfunction in patients with reduced kidney function (as well concomitant diabetes and hypertension), or whether the association reflects more specific interactions between the heart and kidney.

In this retrospective cohort study with available baseline echocardiographic data on a large cohort of patients with and without CKD, we tested the hypothesis that the association of CKD with HFpEF is independent of underlying echocardiographic abnormalities.

\section{Materials and Methods}

\section{Study Population}

This retrospective cohort study enrolled adult patients referred for trans-thoracic echocardiography (TTE) with tissue Doppler imaging at Partners Healthcare-affiliated institutions between January 1st 2004 and January 6th 2014.

Patients $\geq 18$ years old, without history of heart failure, kidney or heart transplant, and with at least one creatinine value during the 365 days before and the 90 days after the TTE were included. Patients who had a first admission for decompensated heart failure prior to 


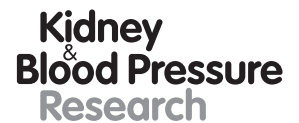

Kidney
Blood Pressure
Research \begin{tabular}{l|l}
\hline Kidney Blood Press Res 2019;44:1247-1258 \\
\hline DOI: 10.1159/000502874 & $\begin{array}{l}\text { @ 2019 The Author(s). Published by S. Karger AG, Basel } \\
\text { www.karger.com/kbr }\end{array}$ \\
\hline
\end{tabular}

Mavrakanas et al.: Association of CKD with HFpEF

the TTE or up to 30 days following the TTE were considered to have heart failure at baseline and were excluded. Average baseline creatinine was calculated for each patient using all available values from 365 days prior to and 90 days after the TTE. Baseline eGFR was calculated using the CKD-EPI equation [14]. Patients were divided into 2 groups: those with preserved renal function defined as eGFR $\geq 90$ (controls), or patients with established CKD, defined as an eGFR $<60 \mathrm{~mL} / \mathrm{min} .1 .73 \mathrm{~m}^{2}$ (cases). Because patients with eGFR between 60 and $89 \mathrm{~mL} / \mathrm{min} .1 .73 \mathrm{~m}^{2}$ do not necessarily fulfill criteria for CKD definition, they were excluded from this study.

\section{Data Collection}

The Research Patient Data registry was used to retrieve all encounters, laboratory tests, medication prescriptions, and TTE results conducted during this time period. It is a centralized clinical data registry gathering clinical information from Partners affiliated healthcare facilities, which includes 5 teaching hospitals.

Information on comorbidities was extracted using condition-specific diagnostic codes (International Classification of Diseases, 9th revision). To be considered as having the respective comorbidities, each patient was required to have a relevant inpatient or outpatient diagnosis prior to the TTE date.

The following laboratory data were extracted using the values closest to the TTE date, within an interval of 365 days before to 90 days after the TTE: urine albumin to creatinine ratios, hemoglobin, albumin, calcium, phosphorus, and brain natriuretic peptide levels.

Medication use was assessed by matching the patients' most recent medication list with an exhaustive list of generic and brand name drugs in these categories: angiotensin converting enzyme inhibitors (ACEIs) or angiotensin receptor blockers (ARBs), $\beta$-blockers, loop diuretics, statins, aspirin, and warfarin. If the medication was prescribed before the date of the TTE, the patient was considered to be taking this at baseline.

All echocardiogram reports were acquired and recorded using an American Society of Echocardiography-recommended scanning protocol with standard techniques. The following parameters were extracted from written reports using automated pattern matching: LVEF, left ventricular diastolic diameter, left ventricular mass index (left ventricular mass corrected for body surface area) (LVMi), left atrium diameter (LAd), pulmonary arterial pressure, regional wall motion abnormalities, right ventricular systolic function (preserved or reduced) and dilatation (presence or absence). Patients with LVEF $\geq 50 \%$ were considered to have preserved ejection fraction. 100 reports were randomly chosen and inspected to confirm the accuracy of the algorithm.

\section{Study Groups and Matching}

The primary analysis (Cohort 1) was designed to study the association of CKD with risk of HFpEF admission. Patients with serial TTEs $(>1)$ and preserved LVEF at baseline were included in this cohort. Cases with CKD were matched 1:1 to controls without CKD on the basis of age ( \pm 5 years), sex, history of hypertension or diabetes, use of ACEIs or ARBs, and $\operatorname{LVEF}( \pm 5 \%)$.

The secondary analysis (Cohort 2) was designed to assess risk of subsequent HFpEF admission in individuals with normal LVMi $\left(\leq 115 \mathrm{~g} / \mathrm{m}^{2}\right.$ for men and $\leq 95 \mathrm{~g} / \mathrm{m}^{2}$ for women $)$ and preserved LVEF at baseline. Cases with CKD were matched 1:1 to controls without CKD for age ( \pm 5 years), sex, history of hypertension or diabetes, and LVEF $( \pm 5 \%)$.

A sensitivity analysis was conducted matching patients in both cohorts only for LVEF at baseline $( \pm 5 \%)$. 


\section{Kidney \\ Blood Pressure \\ Research}

Kidney Blood Press Res 2019;44:1247-1258

Mavrakanas et al.: Association of CKD with HFpEF

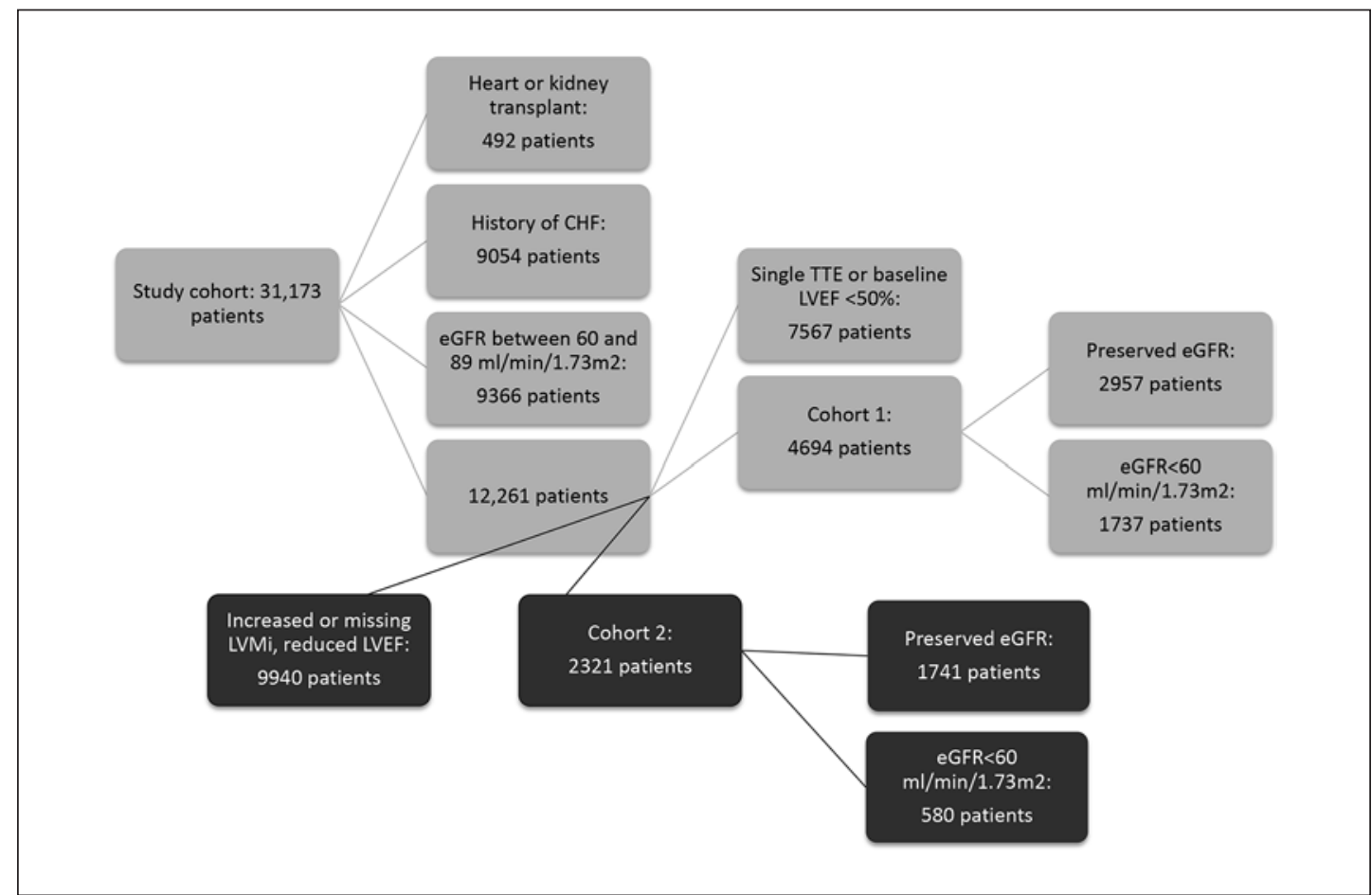

Fig. 1. Flowchart of the study population. Cohort 1 included patients with serial TTEs (trans-thoracic echocardiographies) and preserved LVEF (left ventricular ejection fraction) at baseline. Cohort 2 included patients with normal LVMi (left ventricular mass index) and preserved LVEF at baseline. CHF, congestive heart failure; eGFR, estimated glomerular filtration rate.

\section{Outcomes}

The primary outcome was progression to HFpEF, which was defined as an admission with a diagnostic code for heart failure in patients who had preserved LVEF on the last available TTE. Secondary outcomes were development of any congestive heart failure (CHF) decompensation (regardless of LVEF) and serial changes in echo parameters in patients with and without CKD.

\section{Statistical Analysis}

Results are presented as mean \pm standard deviation, median (interquartile range) or number (\%) for categorical variables. To compare the baseline characteristics, the $\chi^{2}$ test was used for categorical variables, and the Student $t$ test or Mann-Whitney test were used for continuous variables. Standardized differences were calculated to assess balance of the matched cohorts with a cutoff of 0.1 to indicate a negligible difference [15].

Kaplan-Meier plots were constructed and the stratified log-rank test was used to compare the two study groups for the primary and secondary outcomes. Cox proportional hazards regression was used to determine crude and adjusted hazard ratios (HR). Models were adjusted for differences in baseline characteristics of the matched cohorts not directly related to CKD that had remaining discrepancies after matching (CAD, loop diuretics, and LAd for cohort 1 and LAd for cohort 2). Multivariate frailty models were also used to check the impact of matching on outcomes [16].

To better understand the effect of advanced CKD on incidence of HFpEF or any CHF, we conducted an additional sensitivity analysis stratified by an eGFR cutoff of $45 \mathrm{~mL} / \mathrm{min} /$ $1.73 \mathrm{~m}^{2}$. 


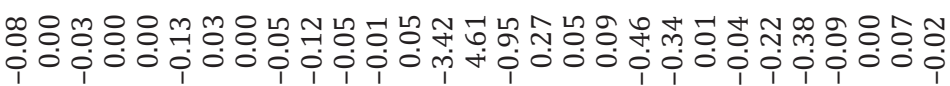

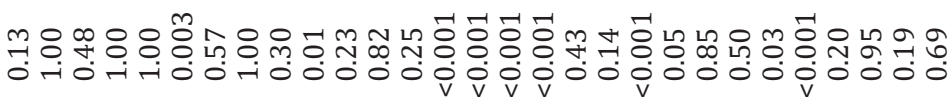

2

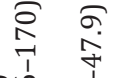

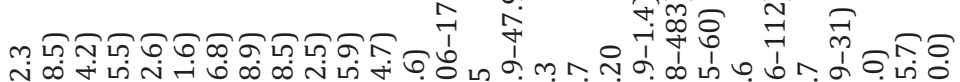

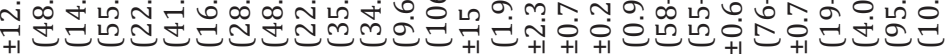

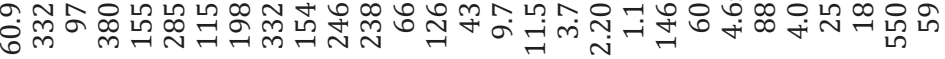

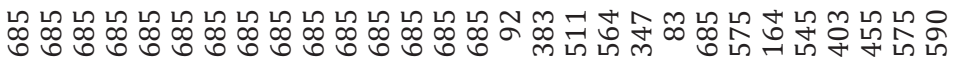

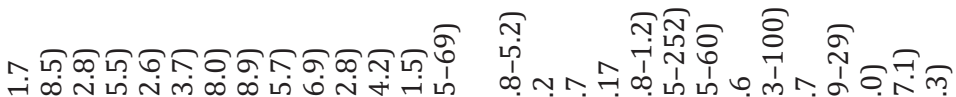
$\exists$ ت 年

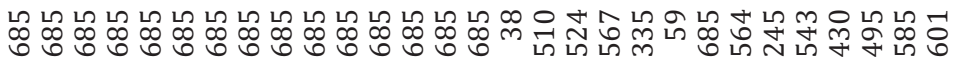

호의

2

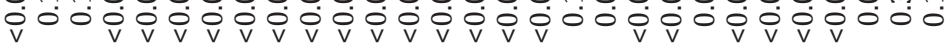

\section{孛}

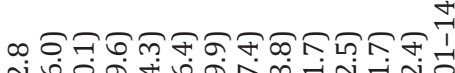

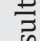

:

空

$\simeq$

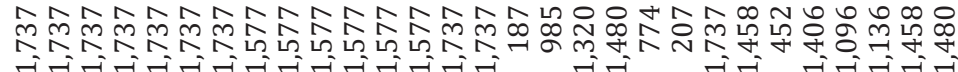

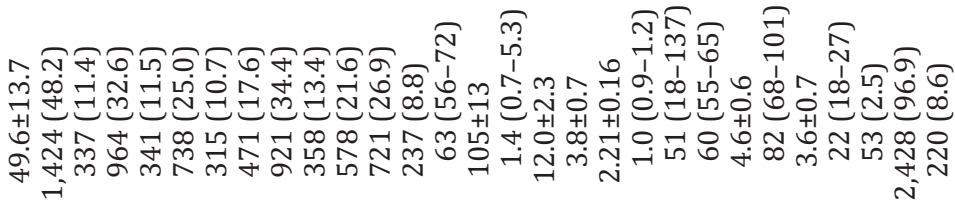

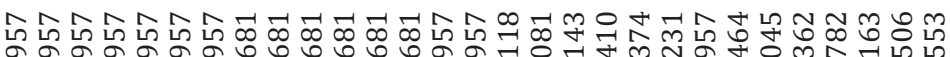

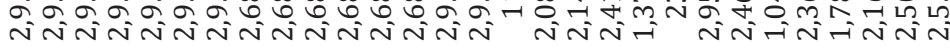

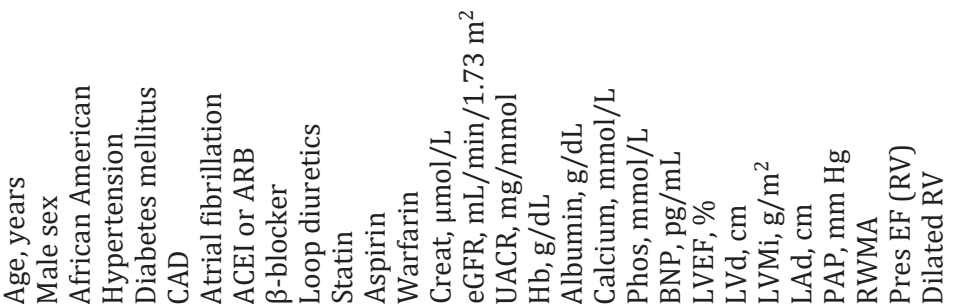

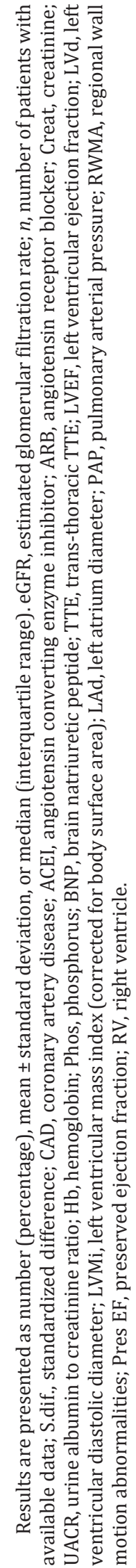




\section{Kidney \\ Blood Pressure \\ Research}

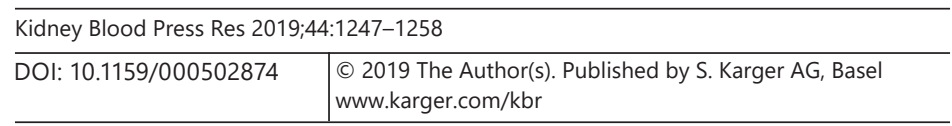

Mavrakanas et al.: Association of CKD with HFpEF

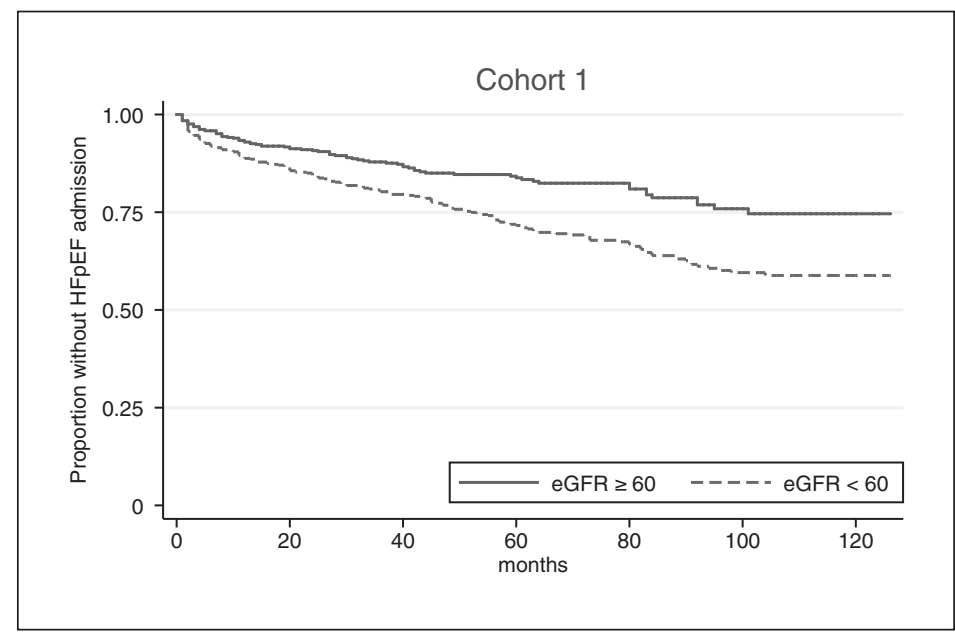

Fig. 2. Kaplan-Meier curves for HFpEF (heart failure with preserved ejection fraction) admission in cohort 1 . Patients with an eGFR (estimated glomerular filtration rate) less than $60 \mathrm{~mL} /$ $\min / 1.73 \mathrm{~m}^{2}$ were at increased risk for HFpEF admission compared with patients with preserved renal function (log rank $p<0.001$, stratified log $\operatorname{rank} p=$ $0.004)$.

To compare the change in echocardiographic parameters during the study in the two groups, a mixed-effects model was used [17]. Patients with at least 3 months interval between the serial TTEs were considered for this analysis.

$p$ values $<0.05$ were considered statistically significant. SPSS (version 20.0, SPSS Inc., Chicago, IL, USA), Stata (version 14 IC; College Station, TX, USA), and SAS (version 9.4, SAS Institute Inc., Cary, NC, USA) were used for the statistical analyses.

\section{Results}

\section{Primary Analysis (Cohort 1)}

The initial cohort comprised 31,173 patients: 492 were excluded due to history of heart or kidney transplant, 9,054 due to CHF, and 9,366 due to an eGFR between 60 and $90 \mathrm{~mL} /$ $\mathrm{min} / 1.73 \mathrm{~m}^{2}$ at baseline (Fig. 1). Of the remaining $12,261,7,567$ patients had only one TTE or an LVEF $<50 \%$ at baseline. Of the remaining 4,694, 2,957 patients had an eGFR $\geq 90 \mathrm{~mL} /$ $\mathrm{min} / 1.73 \mathrm{~m}^{2}$ (control group) and 1,737 patients had an eGFR $<60 \mathrm{~mL} / \mathrm{min} / 1.73 \mathrm{~m}^{2}$ (cases). After matching (1:1) for age ( \pm 5 years), sex, history of hypertension or diabetes, use of ACEIs or ARBs, and LVEF $( \pm 5 \%$ ), the final cohort included 1,370 patients (685 cases and 685 controls). There were 29 dialysis patients in the matched cohort.

Baseline characteristics before and after matching are shown in Table 1. Although significantly different before matching for most characteristics, the two groups were comparable after matching. However, CKD group had higher prevalence of CAD, were more commonly prescribed loop diuretics, and had higher LAd compared with control group, as well as biochemical abnormalities associated with CKD (higher creatinine, higher urine albumin to creatinine ratio, lower $\mathrm{Hb}$, higher phosphorus levels).

A total of 315 patients were admitted for decompensated CHF during follow-up. Among them, LVEF dropped to $<50 \%$ consistent with a diagnosis of heart failure with reduced ejection fraction (HFrEF) in 59 patients, who were excluded from the primary analysis. Patients with CKD were at increased risk for HFpEF admission: crude HR 1.79 (95\% CI 1.38$2.32, p<0.001$ ) and adjusted HR (for CAD, loop diuretics, and LAd) 1.64 (95\% CI 1.22-2.21, $p=0.001$ ). Figure 2 shows the Kaplan Meier curves for admission for HFpEF in the two study groups ( $\log \operatorname{rank} p<0.001$, stratified $\log$ rank $p=0.004)$. Results were similar when all CHF admissions were considered (including patients with LVEF $<50 \%$ in the last available TTE): 
Table 2. Results of multivariate frailty models

\begin{tabular}{llllllll}
\hline Cohort & Outcome & Crude HR & $95 \%$ CI & $p$ & Adjusted HR & $95 \%$ CI & $p$ \\
\hline 1 & HFpEF & 1.79 & $1.38-2.33$ & $<0.001$ & $1.65^{\mathrm{a}}$ & $1.22-2.22$ & 0.001 \\
& Any HF & 1.67 & $1.32-2.11$ & $<0.001$ & $1.48^{\mathrm{a}}$ & $1.13-1.93$ & 0.004 \\
\hline 2 & HFpEF & 1.99 & $1.07-3.71$ & 0.03 & & & \\
& Any HF & & & & $1.70^{\mathrm{b}}$ & $0.98-2.94$ & 0.06 \\
\hline
\end{tabular}

Cells were left blank when frailty models did not converge. HR, hazard ratio; CI, confidence interval; HFpEF, heart failure with preserved ejection fraction; HF, heart failure. ${ }^{a}$ Adjusted for coronary artery disease, loop diuretics use, and left atrial diameter. ${ }^{\mathrm{b}}$ Adjusted for left atrial diameter.

Table 3. Association between chronic kidney disease, stratified by stage, and admission for heart failure

\begin{tabular}{|c|c|c|c|c|}
\hline & \multicolumn{2}{|l|}{ HFpEF } & \multicolumn{2}{|l|}{ Any HF } \\
\hline & HR $(95 \% \mathrm{CI})$ & $p$ & HR (95\% CI) & $p$ \\
\hline \multicolumn{5}{|l|}{ Cohort $1^{\mathrm{a}}$} \\
\hline $\mathrm{eGFR} \geq 90$ & reference & & reference & \\
\hline eGFR 45-60 & $1.43(1.02-2.00)$ & 0.04 & $1.28(0.95-1.73)$ & 0.10 \\
\hline eGFR $<45$ & $1.95(1.39-2.75)$ & $<0.001$ & $1.74(1.28-2.36)$ & $<0.001$ \\
\hline eGFR < 45 vs. $45-60$ & $1.37(0.98-1.90)$ & 0.07 & $1.36(1.00-1.84)$ & 0.048 \\
\hline \multicolumn{5}{|l|}{ Cohort $2^{b}$} \\
\hline $\mathrm{eGFR} \geq 90$ & reference & & reference & \\
\hline eGFR 45-60 & $1.26(0.58-2.72)$ & 0.56 & $1.03(0.52-2.04)$ & 0.93 \\
\hline eGFR $<45$ & $3.28(1.62-6.63)$ & 0.001 & $2.87(1.56-5.26)$ & 0.001 \\
\hline eGFR $<45$ vs. $45-60$ & $2.61(1.25-5.43)$ & 0.01 & $2.78(1.43-5.42)$ & 0.003 \\
\hline
\end{tabular}

HFpEF, heart failure with preserved ejection fraction; HF, heart failure; HR, hazard ratio; CI, confidence interval; eGFR, estimated glomerular filtration rate. ${ }^{\text {a }}$ HRs are adjusted for coronary artery disease, left atrial diameter, and loop diuretics. ${ }^{b}$ HRs are adjusted for left atrial diameter.

crude HR 1.65, 95\% CI 1.31-2.08, $p<0.001$ and adjusted HR (for CAD, loop diuretics, and LAd) 1.47 (95\% CI 1.13-1.92, $p=0.004$ ). Multivariate frailty models yielded similar results (Table 2). More advanced CKD was associated with higher risk for HFpEF and any HF (Table 3).

Because we were able to match only $29 \%$ of patients in our primary analysis, we also performed a sensitivity analysis in which we matched patients with and without CKD on the basis of LVEF $( \pm 5 \%$ ) only. Matching yielded 1427 pairs and results were qualitatively similar (not shown).

In patients with serial TTEs, average time between TTEs was $41 \pm 31$ months (median 34 months, IQR 14-63). LVEF and left ventricular diastolic diameter decreased over time in both patients with and without CKD ( $p<0.001$ and $p<0.001$ respectively), but no difference was observed in rate of dropping between the two groups ( $p=0.39$ and $p=0.83$ respectively). Pulmonary arterial pressure increased over time $(p<0.001)$, at a higher rate in CKD patients $(p=0.008)$.

\section{Secondary Analysis (Cohort 2)}

Of the 12,261 patients qualifying for matching (Fig. 1), 2,321 patients had available LVMi data in the normal range and preserved LVEF at baseline. Of them, 1,741 patients had an eGFR 


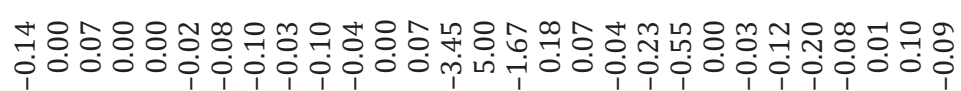

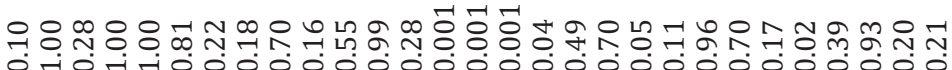

e

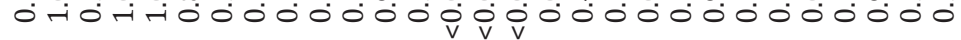

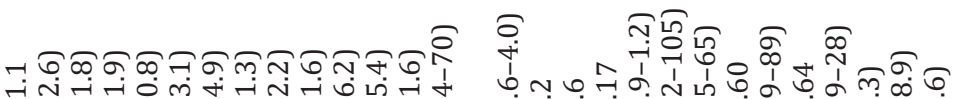

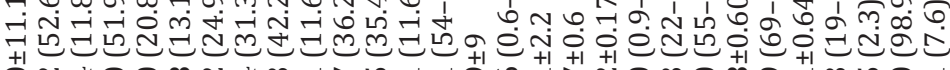

m

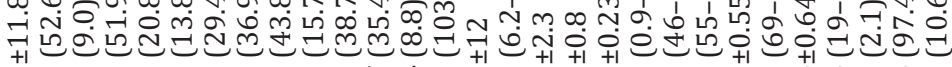

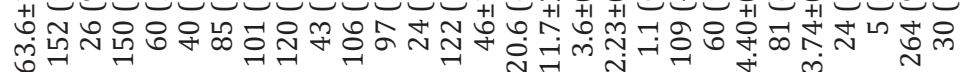

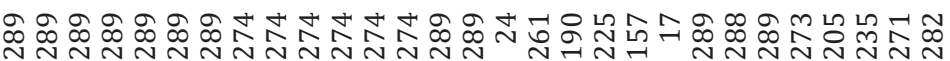
等 N

مి

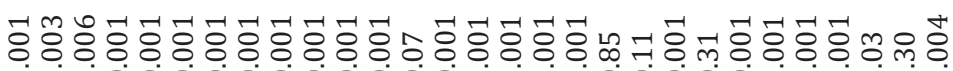
2

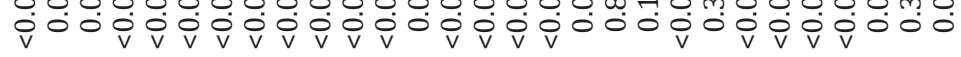

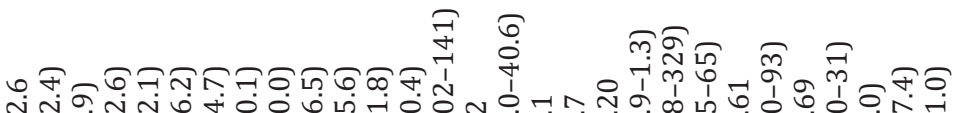

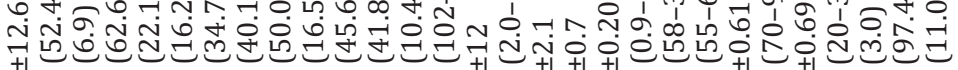

$\pm$

운

o

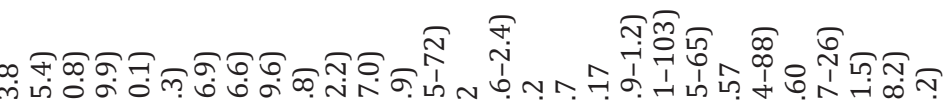
m

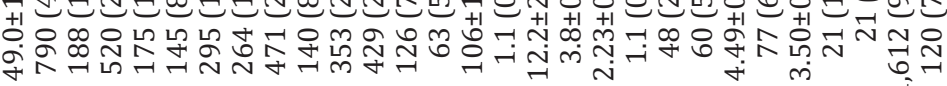

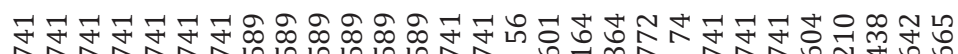
ing

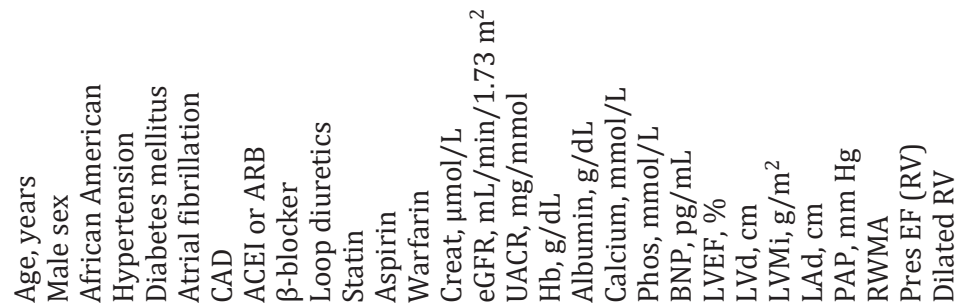

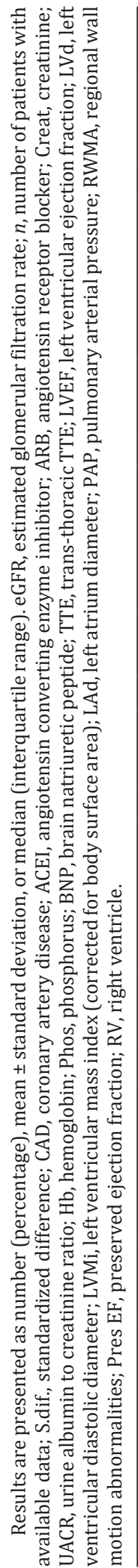




\section{Kidney \\ Blood Pressure \\ Research}

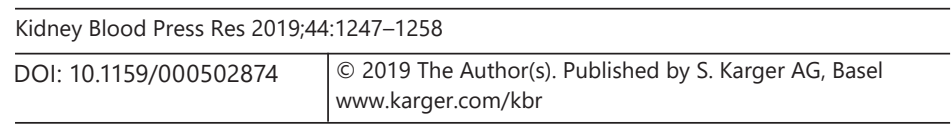

Mavrakanas et al.: Association of CKD with HFpEF

Fig. 3. Kaplan-Meier curves for HFpEF (heart failure with preserved ejection fraction) admission in cohort 2. Patients with an eGFR (estimated glomerular filtration rate) less than $60 \mathrm{~mL} /$ $\min / 1.73 \mathrm{~m}^{2}$ were at increased risk for HFpEF admission compared with patients with preserved renal function (log rank $p=0.03$ ). However, when the stratified log rank test was used to check the impact of matching on outcomes, the difference between the two groups was no longer significant $(p=0.28)$.

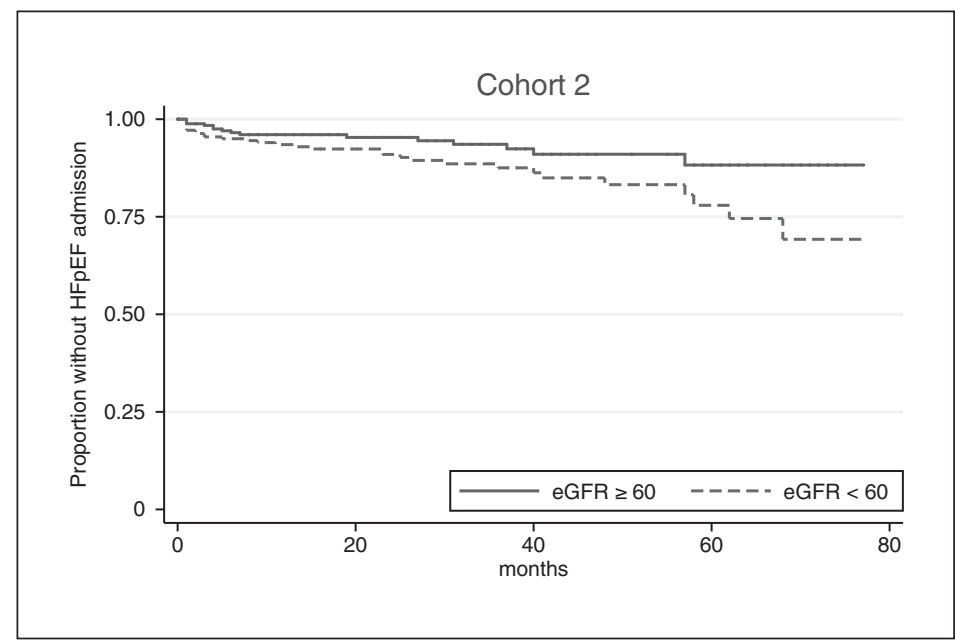

$\geq 90 \mathrm{~mL} / \mathrm{min} / 1.73 \mathrm{~m}^{2}$ (control group) and 580 patients had an eGFR $<60 \mathrm{~mL} / \mathrm{min} / 1.73 \mathrm{~m}^{2}$ (cases). After matching (1:1) for age ( \pm 5 years), sex, history of hypertension or diabetes, and LVEF $( \pm 5 \%)$, the final cohort included 578 patients (289 cases and 289 controls).

Table 4 shows baseline characteristics in those with preserved renal function and with CKD before and after matching. Although significantly different before matching for most of the characteristics, the two groups became comparable after matching. LAd remained higher in CKD patients compared with controls, as did biochemical abnormalities typical of CKD (higher creatinine, higher urine albumin to creatinine ratio, lower Hb levels).

During follow-up, 58 patients were admitted for decompensated heart failure. Among them, LVEF dropped to $<50 \%$ in 14 patients who were excluded from the primary analysis. Patients with CKD were at increased risk for HFpEF admission: crude HR 1.99 (95\% CI 1.07$3.71, p=0.03$ ) and adjusted HR (for LAd) 1.98 (95\% CI 1.05-3.75, $p=0.04$ ). Figure 3 shows the Kaplan Meier curves for admission for HFpEF in the two study groups (log rank $p=0.03$, stratified log rank $p=0.28$ ). Results were similar when all CHF admissions were considered (including patients with $\mathrm{LVEF}<50 \%$ in the last available TTE): crude HR 1.74, 95\% CI 1.022.97, $p=0.04$ and adjusted HR (for LAd) $1.70,95 \%$ CI 0.98-2.94, $p=0.06$. Multivariate frailty models yielded identical results (Table 2). This association was driven by a strong link between kidney function and admission for heart failure in patients with eGFR $<45 \mathrm{~mL} /$ $\min / 1.73 \mathrm{~m}^{2}$ (Table 3).

Because we were able to match only $25 \%$ of patients in our primary analysis, we also matched patients with and without CKD for LVEF ( $\pm 5 \%$ ) only, as a sensitivity analysis. Matching yielded 512 pairs and results were qualitatively similar (not shown).

LVEF decreased over time in patients with and without CKD $(p=0.04)$ but no significant difference was observed in rate of change between the two groups $(p=0.80)$. LVMi and pulmonary arterial pressure increased over time $(p<0.001, p<0.001$ respectively), at a similar rate in patients with and without $\operatorname{CKD~(~} p=0.38, p=0.63$ respectively).

\section{Discussion}

We studied the role of CKD in the development of HFpEF among patients without history of heart failure at baseline after correcting for a full panel of functional and structural cardiac abnormalities. To exclude patients with preexisting asymptomatic cardiac disease, we 


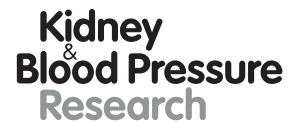

Kidney
Blood Pressure
Research \begin{tabular}{l|l}
\hline Kidney Blood Press Res 2019;44:1247-1258 \\
\hline DOI: 10.1159/000502874 & $\begin{array}{l}\text { @ 2019 The Author(s). Published by S. Karger AG, Basel } \\
\text { www.karger.com/kbr }\end{array}$ \\
\hline
\end{tabular}

Mavrakanas et al.: Association of CKD with HFpEF

designed two cohorts: the first included patients with preserved LVEF, the second only patients with preserved LVEF and normal LVMi at baseline. In an attempt to account for known confounders, we matched for age, sex, history of hypertension and diabetes, and LVEF (as well as for ACEI or ARB use in the first cohort). In both cohorts we found that CKD, defined as an eGFR $<60 \mathrm{~mL} / \mathrm{min} / 1.73 \mathrm{~m}^{2}$, is independently associated with progression to HFpEF.

Our study confirms epidemiological data suggesting a link between CKD and incidence of HFpEF. In the PREVEND cohort, 8,592 patients recruited in the community were prospectively followed for a median of 11.5 years [13]. A total of 374 cases of new-onset heart failure were detected, including 125 cases of HFpEF. Prevalence of CKD, defined as an eGFR (cystatinC based) $<60 \mathrm{~mL} / \mathrm{min} / 1.73 \mathrm{~m}^{2}$, was $12.7 \%$ among heart failure patients versus $6 \%$ among patients who did not develop heart failure. Although CKD prevalence was similar in the HFpEF and HFrEF groups, cystatin $\mathrm{C}$ levels were associated with HFpEF incidence but not with HFrEF. In the Framingham Heart Study, patients with CKD had higher incidence rates of HFpEF and HFrEF compared with individuals with preserved kidney function [18]. CKD was independently associated with heart failure and HFrEF in multivariate Cox models, although this association was attenuated when adjusted for cardiovascular risk factors and valvular heart disease.

These studies either enrolled patients with heart failure or did not account for baseline echocardiographic characteristics and subclinical pre-existing heart disease cannot be excluded in the CKD group. Although they provide compelling proof of an association between $\mathrm{CKD}$ and HFpEF, these studies leave unanswered the question of whether that association is primarily mediated by a high prevalence of structural and functional changes in the heart in CKD - which may in turn reflect the high rate of concomitant diabetes, hypertension and vascular disease in individuals with CKD - or whether this is more directly mediated by changes in kidney function. Although our retrospective cohort cannot definitively answer questions on causality, we expand existing knowledge by showing that HFpEF is more likely to occur in individuals with reduced eGFR than in individuals with preserved kidney function even when baseline cardiac function is similar. We also show that this association is stronger among patients with more advanced CKD $($ eGFR $<45)$. Thus, CKD is independently associated with HFpEF occurrence in individuals with normal baseline TTE characteristics. We show similar trends for any heart failure admission.

If CKD is independently associated with $\mathrm{HFpEF}$, the underlying mechanism is still obscure. CKD and HFpEF share the same risk factors and their joint influence on the kidneys and the heart may explain, at least in part, this observation. However, a distinct pathophysiological link may still be present. In this study, we showed that HFpEF occurs more frequently in CKD patients despite a similar change in TTE parameters over time in both patients with and without CKD. Whether salt retention in the failing kidney is the major factor underlying volume overload in HFpEF remains to be proven [11]. A recent study in a rat model of CKD that develops HFpEF showed impaired cardiomyocyte relaxation associated with dysfunction of the $\mathrm{Na}^{+} / \mathrm{Ca}^{++}$exchanger in nephrectomized rats [19]. eGFR was found to be independently associated with chronotropic incompetence, a common feature in HFpEF [20]. Furthermore, inflammatory and fibrotic processes, both observed in CKD, may be the common link between the two conditions $[21,22]$. Finally, CKD patients have many non-traditional cardiovascular risk factors, such as anemia or mineral bone disease with abnormal calcium and phosphorous levels.

Our study has several limitations. It is a retrospective cohort study of patients referred for TTE with stage 3 or higher CKD. Our results should be generalized cautiously to those with moderate CKD or more broadly to patients who would not be referred for echocardiographic monitoring in routine practice. Further, TTEs were interpreted by multiple clinical cardiologists rather than by a core lab and were done to answer a specific clinical question and not to 
provide systematic information on specific cardiac function and structure variables. Lastly, tissue Doppler imaging results were not extractable for most patients in the registry and diastolic function could not be assessed. Diagnostic codes were used for comorbidities and clinical outcomes; no separate adjudication of the outcomes was performed. Follow-up in a large, dedicated echocardiographic research study using a blinded, core-reading center should be considered to confirm our findings. Nevertheless, the strengths of this study, including baseline and longitudinal echocardiographic data, matching for several well recognized confounders, and assessment of multiple echocardiographic parameters represent unique strengths of our analysis and help provide important new information.

\section{Conclusion}

In conclusion, CKD is independently associated with new onset HFpEF. Future research should focus on the emerging pathophysiological pathways common in both disorders in order to clarify the link between CKD and HFpEF and to identify potential treatment targets.

\section{Acknowledgments}

This work was presented in the form of a poster abstract at the 2017 Kidney Week of the American Society of Nephrology in New Orleans.

This work was conducted with support from Harvard Catalyst | The Harvard Clinical and Translational Science Center (National Center for Research Resources and the National Center for Advancing Translational Sciences, National Institutes of Health Award UL1 TR001102) and financial contributions from Harvard University and its affiliated academic healthcare centers. The content is solely the responsibility of the authors and does not necessarily represent the official views of Harvard Catalyst, Harvard University and its affiliated academic healthcare centers, or the National Institutes of Health.

This work was supported by a National Institute of Diabetes and Digestive and Kidney Diseases training grant for Drs. Khattak and Singh [T32DK007527].

Dr. Mavrakanas was supported by a grant from the foundation G. and L. Hirsch (General Internal Medicine Division, Geneva University Hospitals).

\section{Statement of Ethics}

The Partners Healthcare institutional review board approved this study but waived the need to obtain consent from participants. The study conforms to the ethical standards of the Declaration of Helsinki.

\section{Disclosure Statement}

The authors have no conflicts of interest to disclose. 
Mavrakanas et al.: Association of CKD with HFpEF

\section{References}

1 Paulus WJ, Tschöpe C, Sanderson JE, Rusconi C, Flachskampf FA, Rademakers FE, et al. How to diagnose diastolic heart failure: a consensus statement on the diagnosis of heart failure with normal left ventricular ejection fraction by the Heart Failure and Echocardiography Associations of the European Society of Cardiology. Eur Heart J. 2007 Oct;28(20):2539-50.

2 Redfield MM, Jacobsen SJ, Burnett JC Jr, Mahoney DW, Bailey KR, Rodeheffer RJ. Burden of systolic and diastolic ventricular dysfunction in the community: appreciating the scope of the heart failure epidemic. JAMA. 2003 Jan;289(2):194-202.

3 Vasan RS, Levy D. The role of hypertension in the pathogenesis of heart failure. A clinical mechanistic overview. Arch Intern Med. 1996 Sep;156(16):1789-96.

4 Hwang SJ, Melenovsky V, Borlaug BA. Implications of coronary artery disease in heart failure with preserved ejection fraction. J Am Coll Cardiol. 2014 Jul;63(25 25 Pt A):2817-27.

5 Redfield MM. Heart failure with preserved ejection fraction. N Engl J Med. 2016 Nov;375(19):1868-77.

6 Paulus WJ, Tschöpe C. A novel paradigm for heart failure with preserved ejection fraction: comorbidities drive myocardial dysfunction and remodeling through coronary microvascular endothelial inflammation. J Am Coll Cardiol. 2013 Jul;62(4):263-71.

7 Mohammed SF, Hussain S, Mirzoyev SA, Edwards WD, Maleszewski JJ, Redfield MM. Coronary microvascular rarefaction and myocardial fibrosis in heart failure with preserved ejection fraction. Circulation. 2015 Feb; 131(6):550-9.

8 Unger ED, Dubin RF, Deo R, Daruwalla V, Friedman JL, Medina C, et al. Association of chronic kidney disease with abnormal cardiac mechanics and adverse outcomes in patients with heart failure and preserved ejection fraction. Eur J Heart Fail. 2016 Jan;18(1):103-12.

9 Smith DH, Thorp ML, Gurwitz JH, McManus DD, Goldberg RJ, Allen LA, et al. Chronic kidney disease and outcomes in heart failure with preserved versus reduced ejection fraction: the Cardiovascular Research Network PRESERVE Study. Circ Cardiovasc Qual Outcomes. 2013 May;6(3):333-42.

10 Löfman I, Szummer K, Dahlström U, Jernberg T, Lund LH. Associations with and prognostic impact of chronic kidney disease in heart failure with preserved, mid-range, and reduced ejection fraction. Eur J Heart Fail. 2017 Dec;19(12):1606-14.

11 Fang JC. Heart Failure With Preserved Ejection Fraction: A Kidney Disorder? Circulation. 2016 Aug;134(6): 435-7.

12 Waheed S, Matsushita K, Sang Y, Hoogeveen R, Ballantyne C, Coresh J, et al. Combined association of albuminuria and cystatin C-based estimated GFR with mortality, coronary heart disease, and heart failure outcomes: the Atherosclerosis Risk in Communities (ARIC) Study. Am J Kidney Dis. 2012 Aug;60(2):207-16.

13 Brouwers FP, de Boer RA, van der Harst P, Voors AA, Gansevoort RT, Bakker SJ, et al. Incidence and epidemiology of new onset heart failure with preserved vs. reduced ejection fraction in a community-based cohort: 11-year follow-up of PREVEND. Eur Heart J. 2013 May;34(19):1424-31.

14 Levey AS, Stevens LA, Schmid CH, Zhang YL, Castro AF 3rd, Feldman HI, et al.; CKD-EPI (Chronic Kidney Disease Epidemiology Collaboration). A new equation to estimate glomerular filtration rate. Ann Intern Med. 2009 May;150(9):604-12.

15 Normand ST, Landrum MB, Guadagnoli E, Ayanian JZ, Ryan TJ, Cleary PD, et al. Validating recommendations for coronary angiography following acute myocardial infarction in the elderly: a matched analysis using propensity scores. J Clin Epidemiol. 2001 Apr;54(4):387-98.

16 Vaupel JW, Manton KG, Stallard E. The impact of heterogeneity in individual frailty on the dynamics of mortality. Demography. 1979 Aug;16(3):439-54.

17 Laird NM, Ware JH. Random-effects models for longitudinal data. Biometrics. 1982 Dec;38(4):963-74.

18 Nayor M, Larson MG, Wang N, Santhanakrishnan R, Lee DS, Tsao CW, et al. The association of chronic kidney disease and microalbuminuria with heart failure with preserved vs. reduced ejection fraction. Eur J Heart Fail. 2017 May;19(5):615-23.

19 Primessnig U, Schönleitner P, Höll A, Pfeiffer S, Bracic T, Rau T, et al. Novel pathomechanisms of cardiomyocyte dysfunction in a model of heart failure with preserved ejection fraction. Eur J Heart Fail. 2016 Aug;18(8): 987-97.

20 Klein DA, Katz DH, Beussink-Nelson L, Sanchez CL, Strzelczyk TA, Shah SJ. Association of chronic kidney disease with chronotropic incompetence in heart failure with preserved ejection fraction. Am J Cardiol. 2015 Oct;116(7):1093-100.

21 Betjes MG. Immune cell dysfunction and inflammation in end-stage renal disease. Nat Rev Nephrol. 2013 May; 9(5):255-65.

22 Liu Y. Cellular and molecular mechanisms of renal fibrosis. Nat Rev Nephrol. 2011 Oct; 7(12):684-96. 